\title{
OP72
}

\section{OPTIMIZATION OF ROAD SURFACE REFLECTIONS PROPERTIES AND LIGHTING: LEARNING OF A THREE- YEAR EXPERIMENT}

\author{
Valérie Muzet et al.
}

DOI 10.25039/x46.2019.OP72

from

CIE x046:2019

Proceedings

of the

29th CIE SESSION

Washington D.C., USA, June 14 - 22, 2019

(DOI 10.25039/x46.2019)

The paper has been presented at the 29th CIE Session, Washington D.C., USA, June 14-22, 2019. It has not been peer-reviewed by CIE.

(c) CIE 2019

All rights reserved. Unless otherwise specified, no part of this publication may be reproduced or utilized in any form or by any means, electronic or mechanical, including photocopying and microfilm, without permission in writing from CIE Central Bureau at the address below. Any mention of organizations or products does not imply endorsement by the CIE.

This paper is made available open access for individual use. However, in all other cases all rights are reserved unless explicit permission is sought from and given by the CIE.

CIE Central Bureau

Babenbergerstrasse 9

A-1010 Vienna

Austria

Tel.: +4317143187

e-mail: ciecb@cie.co.at

www.cie.co.at 


\title{
OPTIMIZATION OF ROAD SURFACE REFLECTIONS PROPERTIES AND LIGHTING: LEARNING OF A THREE-YEAR EXPERIMENT
}

\author{
Muzet, V. ${ }^{1}$, Greffier F. ${ }^{2}$, Verny P. ${ }^{3}$ \\ ${ }^{1}$ Cerema, Equipe projet ENDSUM, Strasbourg, FRANCE \\ ${ }^{2}$ Cerema, Angers, FRANCE, \\ ${ }^{3}$ Cerema, Aix, FRANCE \\ valerie.muzet@cerema.fr
}

DOI $10.25039 / \times 46.2019 .0 P 72$

\begin{abstract}
Adapting light distributions of luminaires to different types of road surfaces when new and also along their lifetime remains a challenge. In this paper, we present the assessment, over its first three years, of an innovative compared to a conventional infrastructure renovation. The innovative solution, called Lumiroute ${ }^{\circledR}$, integrates both a light road surface and LED lighting that can be tuned to follow the evolution of the road surface photometry. Regular on-site evaluation of the conventional and photometric characteristics of the pavements and the luminance perceived by the driver over time were performed. This paper presents the outcome of this experiment, in terms of photometry, energy consumption, obtrusive light and financial aspects of the project.
\end{abstract}

Keywords: Road photometry, Adaptative road lighting, Real-site experiment, Time evolution, Obtrusive light

\section{Introduction}

Road lighting installations of interurban roads are designed by calculating the performance in terms of luminance distribution as defined in the EN 13201 standard (CEN, 2015). In a context of energy saving and obtrusive light reduction, it is important to consider both the photometric characteristics of the luminaires and the road. But taking into account the actual photometric characteristics of a road surface to design and then adjust a lighting installation is seldom done. Since the photometric characteristics of the pavements are generally not measured, a reference $r$-table as defined in (CIE, 1984); (CIE, 2001) is often used for lighting design and the changes of the photometry over time are completely ignored. However, the road surface is a key element in lighting calculations and several studies have shown that the CIE reference r-tables are no longer representative of actual pavements (Dumont, 2007a); (Dumont et al., 2007b); (Jacket et al., 2010); (Ylinen et al., 2010); (Petrinska et al., 2007); (Muzet et al., 2017); (Muzet et al., 2018). Their use can generate important errors (more than $30 \%$ ) for the average luminance (Chain et al., 2007).

An innovative concept, called Lumiroute ${ }$, was proposed by a civil engineering company ${ }^{1}$ and a lighting designer2. The rational is to integrate the photometry of a light pavement in an adaptive LED lighting solution. The renovation of a suburban road in Limoges, France, made it possible to implement the concept in real conditions (Chain et al., 2015). The Cerema has been commissioned by the French $\mathrm{CIRR}^{3}$ for the experimentation framework and to assess the innovative solution, in comparison with a conventional renovation, during the first 3 years of its lifetime. More specifically:

- The "conventional" projects use two ordinary pavements (a raw VTAC4 and a water jet scrubbed VTAC), a metal halide lamp and classic lighting design (using CIE r-table R3).

\footnotetext{
1 Malet-Spie Batignolles

2 Zumtobel Group Lighting France

3 Innovative Comity of Roads and Streets

4 Very Thin Asphalt Concrete
} 
- The innovative Lumiroute ${ }^{\circledR}$ project uses two light pavements (a raw VTAC with light coloured aggregates, synthetic binder and adding of $\mathrm{TiO}_{2}$ and a water jet scrubbed VTAC with light coloured aggregates), adjustable LED lamps and optimised lighting design using the measured photometry of the pavements.

Our contribution concerns the on-site evaluation of the photometric characteristics of the pavements and the luminance perceived by the driver over time, but also the energy consumption, obtrusive light and financial feasibility of the project.

The paper is organised as follows. In the first part, we recall the basics of road lighting obtrusive light. Then, we describe the experimental site and the evaluation protocol. In the second part, the results of the study regarding road surface photometry, luminance and obtrusive light are given and discussed. The last part contains an economic evaluation taking into account energy assessment, investment and operating costs.

\section{Experimental setup and methodology}

\subsection{Road lighting basics}

Guidelines (CIE, 2001); (CIE, 2019) and road lighting standards in Europe (CEN, 2015b \& 2015c) give threshold minimum values for illuminance and luminance and their distribution on the road surface according to a grid of points whose number $N$ depends on the pole spacing $S$ and of the number of traffic lanes (Figure 1a). The average luminance $\left(L_{\text {ave }}\right)$, the overall luminance uniformity ratio $\left(U_{0}\right)$ and longitudinal luminance uniformity ratio $\left(U_{1}\right)$ are computed according to standard specifications.
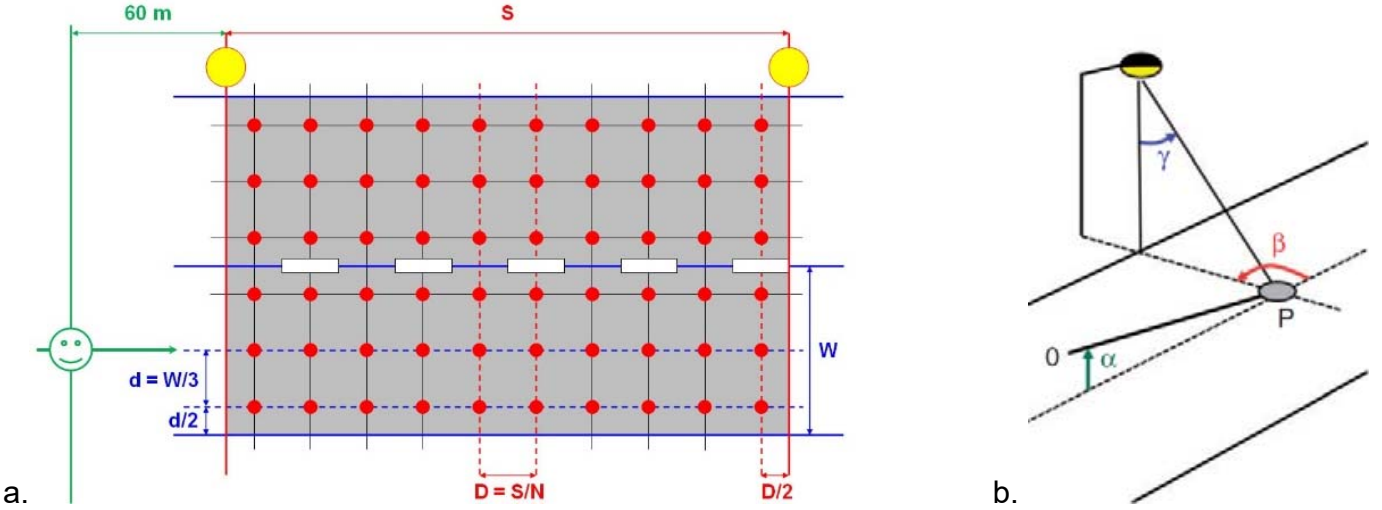

Figure 1 - a. Example of a calculation /measurement grid points in road lighting design/evaluation. :- represents the eye of the driver, $W$ the traffic lane width, $d$ the transversal distance between two lines of points and $D$ the longitudinal distance between two columns.

b. The photometric characteristics of the road surface depend on the angles of observation $\alpha$, sight $\beta$ and incidence $\gamma$. The origin " 0 " represents the driver and P the point of observation.

The reflection properties of the road surface are used for the computation of a lighting installation. The reduced luminance coefficient $r$ is defined as:

$$
r(\beta, \gamma)=10^{4} \cdot L(\beta, \gamma) / E_{\mathrm{h}} \cdot \cos ^{3} \gamma=10^{4} \cdot q(\beta, \gamma) \cdot \cos ^{3} \gamma,
$$

where,

$L$ is the observed luminance in $\mathrm{cd} \cdot \mathrm{m}^{-2}$,

$E_{\mathrm{h}}$ the horizontal illuminance in lux,

$q$ the reflection coefficient in $c d . m^{-2}$.lux ${ }^{-1}$.

The angle of observation $\alpha$ is set at $1^{\circ}$, which corresponds to a driver looking at about $90 \mathrm{~m}$ (CIE, 2001). The $r$-table is a two-dimensional table with a number of standardized combinations of the incidence lighting angle $\gamma$ and orientation angle $\beta$, the boundaries of which define a solid angle $\Omega$ (Figure $1 \mathrm{~b}$ ). To simplify the description of photometric performance of road surfaces, the additional parameters $Q_{0}$ and $S_{1}$ can be calculated from the previous matrix as following: 


$$
Q_{0}=\int_{0}^{\Omega} \frac{q(\beta, \tan \gamma) \cdot d \Omega}{\Omega} .
$$

$Q_{0}$ is the solid angle weighted average of all the luminance coefficients also called lightness (van Bommel, 2015) and the specularity factor $S_{1}$ (also called shininess), is given by:

$$
S_{1}=\frac{r(\beta=0, \tan \gamma=2)}{r(\beta=0, \tan \gamma=0)} .
$$

The evaluations of the contribution of non-useful or potentially harmful light from lighting installations are carried out on the basis on the CIE150-2017 technical report (CIE150, 2017) that defines several representative quantities in its annex D:

- the maximum upward luminous flux potentially lost towards the sky called UPF $\max$

$$
\Phi \cup \mathrm{P}, \max =\Phi \mathrm{la}\left[R \cup L O, \alpha+\rho_{1} u+\rho_{2}(R \mathrm{DLO}, \alpha-u)\right]
$$

where,

$\Phi_{l a}$ is the assigned luminous flux of all involved lamps;

$R \cup L O, \alpha$ is the upward light output ratio of the installation for the luminaire tilting angle $\alpha ; R_{\mathrm{DLO}, \alpha}$ is the downward light output ratio of an installation for the tilting angle $\alpha$;

$\rho_{1}$ is the average reflectance of the reference surface and $\rho_{2}$ the surroundings one;

$u$ is the utilization factor of the installation for the reference surface $S$ whose area is $A$.

- the minimum value of $U P F_{\min }$ (symbol $\Phi \cup P, \min$ ) is the irreducible minimum flow reflected towards the sky reflected by the reference lighted surface and is defined by

$$
\Phi \cup P, \min =\rho_{1} E_{\mathrm{m}} A
$$

where $E_{\mathrm{m}}$ is the average maintenance illuminance.

- the Upward Flux Ratio UFR or RuL is the ratio of $\Phi \cup P, \max$ and $\Phi \cup P, \min$.

In order to calculate the upward light parameters, it is necessary to know the average reflectance of the road surface $\rho_{1}$. It is possible to use values based on old measurements from 1999 (CIE150, 2017 annex E) or to measure it:

- on samples according to CIE093 (CIE, 1993) with

$$
r=\pi \cdot L / E \text {, }
$$

where $L$ is the average luminance of the surface and $E$ the average illuminance of the surface,

- on the ground surface with an illuminance meter as specified in CIE150-2017 annex E

$$
r=E r / E i \text {, }
$$

where, $E \mathrm{i}$ is the average illuminance of the surface,

$\mathrm{Er}$ is measured at $1 \mathrm{~m}$ with the illuminancemeter looking at the ground.

\subsection{The experimental setup and initial design}

The experimental site is a $2 \times 2$ lane pavement 6.50 metres wide and 400 metres long, located on a suburban road (Boulevard Schumann) in Limoges, France. The speed limit is $70 \mathrm{~km} / \mathrm{h}$ with a traffic between 150 and 300 trucks per day. The 4 metres wide central reserve is equipped with a 9 metres high lighting system with twin central arrangement. The road surfacing of the entire Boulevard Schuman is a VTAC with a $0 / 10 \mathrm{~mm}$ gradation. The experimental zone consists of two sections of 200 metres on either side of the boulevard (Figure 2). Section 1 and 2 are the innovative sections called Lumiroute $₫$ sections and section 3 and 4 are ordinary control sections. Sections 1 and 4 are raw sections while the surfaces of sections 2 and 3 are treated with high-pressure water jet to remove the thin bituminous layer from the surface. Due to the close location of all the tested sections, environmental conditions such as aging, traffic, meteorological conditions were the same, enabling comparisons between the different sections. 



Figure 2 - a. Framework of the implementation of the 4 sections, b. Picture of the 4 pavements.

The lighting installation was designed in 2013 by Zumtobel with Lighting Reality software, using the theoretical normative values (CEN, 2003) of the lighting class ME3a (Lave=1.00 $\mathrm{cd} / \mathrm{m}^{2}$; $U_{0}=0.40 ; U_{1}=0.70$ ), taking into account the maintenance factor. For the "control sections" 3 and 4 composed of ordinary road surfaces and metal halide lighting, a typical lighting calculation was done using the R3 CIE $r$-table $\left(Q_{0}=0.07, S_{1}=1.1\right)$ without rescaling. For the two Lumiroute ${ }^{\circledR}$ sections composed of light surfaces and adjustable LED lamps, the design was done with the $r$-table measured with the Cerema laboratory goniophotometer (Paumier, 2006), on specifically built samples of the two chosen formulations. Lumiroute $\AA$ section 1 is a class R2 pavement $\left(Q_{0}=0.133, S_{1}=0.74\right)$ and Lumiroute $\circledast$ section 2 a class $R 1$ pavement $\left(Q_{0}=0.074, S_{1}=0.40\right)$.

The resulting 4 combinations of road surface and lighting are summarized below:

- Section 1 is composed of the Lumiroute $\circledast 1$ pavement (raw surface with light-colored aggregates, a synthetic binder and white pigment $\mathrm{TiO}_{2}$ ) combined with a LED illumination of $77 \mathrm{~W}$ (STYLED lamp, color temperature $4000 \mathrm{~K}$ ).

- Section 2 consists of Lumiroute ${ }^{\circledR} 2$ pavement (water jet scrubbed pavement with light aggregates and a bituminous binder) combined with a LED illumination of $103 \mathrm{~W}$ (STYLED lamp, color temperature $4000 \mathrm{~K}$ ).

- Section 3 is composed of a "Control treated" road surface (water jet scrubbed pavement with grey aggregate and a bituminous binder) combined with traditional lighting consisting of a $140 \mathrm{~W}$ metal halide discharge source (COSMO lamp, color temperature of $2811 \mathrm{~K}$ ).

- Section 4 is composed of a "Control raw" road surface (current pavement with grey aggregate and a bituminous binder) combined with the same $140 \mathrm{~W}$ metal halide discharge source.

In all sections, a $40 \%$ power reduction was applied during the off-peak hours of night-time traffic. On the Lumiroute ${ }^{\circledR}$ sections, DALI (Digital Adressable Lighting Interface) was used to enable adaptations of the LED lighting intensity when needed.

\subsection{Evaluation protocol}

The study was conducted between December 2013 and September 2017. The monitoring included regular evaluations every six months:

- Conventional evaluation of the road pavements was done every to check whether the innovative formulations had any drawbacks, especially concerning safety issues.

- Photometric on-site evaluation of pavement with a portable measurement device named COLUROUTE (see picture in Figure 3a). This transportable measuring device complies with the specifications of the CIE with a measurement at an observation angle of $1^{\circ}$. It is composed of 27 light sources corresponding to combinations of angles of the $r$-table and in particular those used for the determination of $S_{1}$ (Muzet et al., 2007). The $r$-table is reconstituted and the coefficient $Q_{0}$ calculated. It enables the photometric characteristics of pavements to be monitored over time (Muzet et al., 2017).

- Assessment of lighting quality and compliance with normative requirements. For these luminance measurements, an automatic mobile ILMD (Imaging Luminance Measuring Device) called CYCLOPE system has been used (Greffier et al., 2015). The normative measurement grid for performance calculation (Figure 1a) is projected onto the images by using road marking detection and robust fitting. From these luminance values, 
average luminance, overall uniformity and longitudinal uniformity are calculated. Based on these measurements, tuning of the lighting installations of the innovative sections could be conducted.

- Evaluations of the contribution of non-useful or potentially harmful light from lighting installations carried.

- Economic analysis with energy assessment, investment and operating costs.

\section{Results and discussions}

\subsection{Conventional evaluation of the road pavements}

Conventional evaluation of the road pavements consisted in regular measurements of skid resistance, pneumatic contact noise of the pavement and search for degradation with aging. The measured sound levels were stable and similar on the four sections with an average value of $95.8 \mathrm{~dB}(\mathrm{~A})$ which is typical for a VTAC $0 / 10$. The macrotexture was measured according to the CEN 13036-1 (CEN, 2010). There was a slight increase in the macrotexture of the four pavements with time. The skid resistance was assessed according to CEN 15901-6 (CEN, 2009). Despite a reduction in the average skid resistance over time on all the sections, the measured Transversal Friction Coefficient (TCR) was between 55 and 71 at the end of the experiment, which is a sufficient skid resistance in France (the limit is 50). In addition, as no significant rutting was detected after the 3 years, it was demonstrated that the performance of all the section was normal for the four types of pavements.

\subsection{Road surface photometry}

At each measurement campaign, there was 8 characterizations per section with the COLUROUTE portable gonioreflectometer (Figure 3a). The average and standard deviation of these measures were calculated for $Q_{0}$ and $S_{1}$ and are shown in the Table 1 . The raw sections 1 and 4 , initially very specular were particularly heterogeneous at the beginning of the experiment, whereas the treated surfaces (Sections 2 and 3 ) were quite homogenous. After 3 years of circulation, the dispersion of measurements of the 4 sections was low, whether for lightness $Q_{0}$ or specularity $S_{1}$, which shows that a stable state has been reached.

The specularity difference between the water jet scrubbed sections (Sections 2 and 3 ) and the other two (Section 1 and 4) has faded over time. In fact, the specularity of sections 1 and 4 , which were initially not treated, dropped sharply in 3 years. The specularity of the treated sections 2 and 3 decreased slightly over time. After 3 years of circulation, the specularity of the raw section 4 remained higher than the other sections.

The lightness coefficient $Q_{0}$ increased over time for the control section 4 and decreased for section 1, Lumiroute $\AA$ 1. For the water jet scrubbed surfaces Lumiroute ${ }^{2} 2$ and section 3 , Q0 increased. After 3 years, the Lumiroute ${ }^{\circledR} 1$ and 2 pavements have a lightness coefficient $Q_{0}$ greater (between 0.9 and 0.11 ) than the ordinary sections 3 and 4 (between 0.6 and 0.8 ). So these sections, initially selected for their ability to reflect more light remain interesting after the impact of aging.

Table 1 - On-site photometric measurements with COLUROUTE device between T0 and 36 months. Evolution over time of average values of $Q_{0}, S_{1}$ and corresponding standard deviation.

\begin{tabular}{|c|c|c|c|c|c|c|c|c|c|c|c|c|c|}
\hline & \multicolumn{2}{|c|}{ T0 month } & \multicolumn{2}{|c|}{ T6 month } & \multicolumn{2}{|c|}{ T12 month } & \multicolumn{2}{|c|}{ T24 month } & \multicolumn{2}{|c|}{ T30 month } & \multicolumn{2}{|c|}{ T36 month } \\
\hline & & Mean & Std & ean & Std & lean & Std & Mean & Std & nean & Std & Mean & Std \\
\hline \multirow{2}{*}{$\begin{array}{c}\text { S1: } \\
\text { Lumiroute1 }\end{array}$} & $Q_{0}$ & 5 & 0 & 8 & 0.011 & & 0. & & 0.006 & & 0.009 & 102 & .008 \\
\hline & 91 & & 21 & 4 & 26 & & 0.17 & 6 & .12 & & 0.20 & 5 & 06 \\
\hline \multirow{2}{*}{$\begin{array}{c}\text { S2: } \\
\text { Lumiroute2 }\end{array}$} & & & & & c & & 9 & & 8 & & 08 & & 12 \\
\hline & $S$ & & & & & & 0.06 & & 8 & & 5 & & \\
\hline \multirow{2}{*}{$\begin{array}{l}\text { S3: Control } \\
\text { Treated }\end{array}$} & $Q_{0}$ & 0 & مـ & 8 & 0.0 & & 0.006 & & 0.003 & & 0.003 & & 005 \\
\hline & 5 & & & & 0 & & .07 & & 6 & & 11 & & 6 \\
\hline \multirow{2}{*}{$\begin{array}{l}\text { S4: Control } \\
\text { Raw }\end{array}$} & $Q_{0}$ & 0.0 & 0.0 & 2 & 0.004 & & 0.007 & 0.065 & 0.004 & & 0.004 & .070 & 0.008 \\
\hline & S1 & 3.01 & 0.75 & 1.32 & 0.18 & 0.98 & 0.14 & 0.86 & 0.16 & 0.84 & 0.18 & 0.96 & 0.19 \\
\hline
\end{tabular}


a.
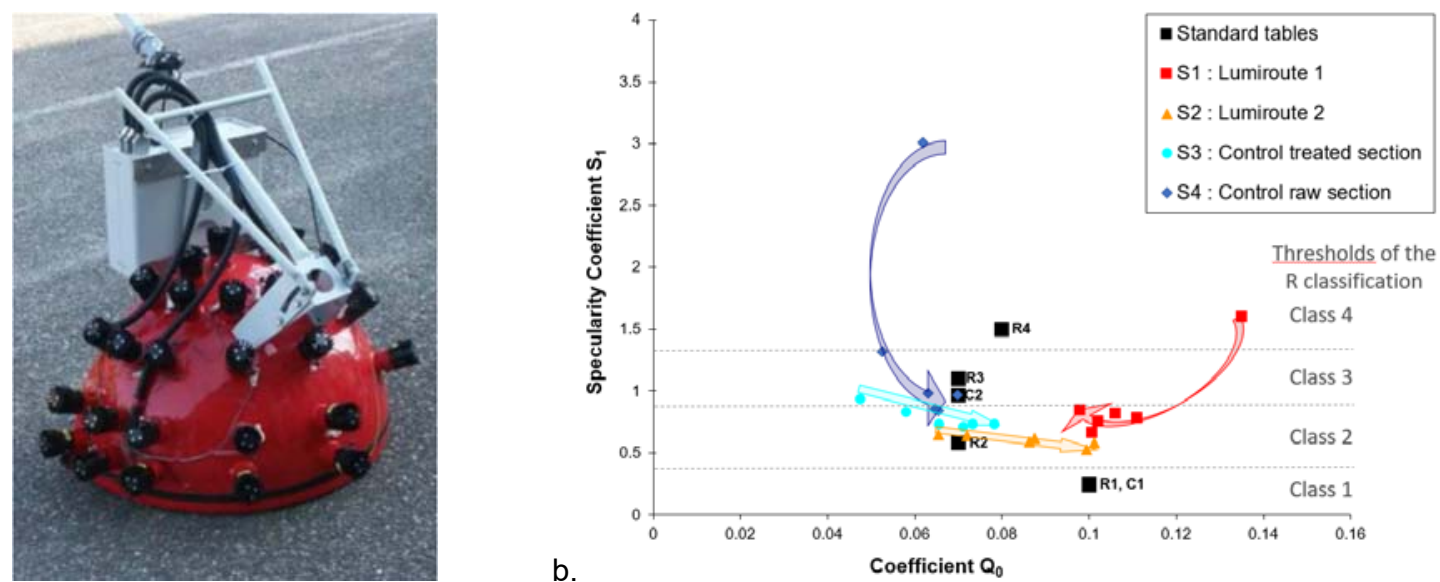

Figure 3 - a. Picture of COLUROUTE device, b. Average values of the photometric characteristics of the four sections during the three years of the study. The arrows represent the evolution of each sections with time.

As represented in the Figure $3 b$, some of our $Q_{0}$ and $S_{1}$ measurements differ from the photometric characteristics of the typical CIE r-tables R or C (CIE, 2001), especially when the pavements are new.

For the initially raw surfaces Lumiroute ${ }^{\circledR} 1$ and section 4 , the observed evolution of the photometry of pavement is in accordance with a French previous study (Dumont et al., 2007b), especially for the important decrease of specularity and the darkening of light sections. In this previous study, the photometry of road surfaces was followed during 3 years with regular extraction of cores measured in laboratory but there was no investigation on water jet scrubbed sections. The water jet scrubbed sections 2 and 3 offer interesting opportunities from the beginning because they have an initial homogeneity with a low specularity. Moreover, the observed increase of $Q_{0}$ with time is interesting because it could be a compensation of lamp aging.

\subsection{Luminance and uniformity}

The conformity assessment of the 2003 standard (CEN, 2003) was carried out both in static and dynamic conditions with the CYCLOPE system, measuring the photometric parameters: $L_{\text {ave, }} U_{0}$ and $U_{1}$, for all sections, over their entire length.

For optimized lighting, the Lumiroute ${ }^{\circledR}$ process in sections 1 and 2 allow the tuning of the luminous flux. In accordance with the evaluation protocol, no power adjustment was done on the two control sections, so sections 3 and 4 remained unchanged. After the analysis of the photometric parameters of section 1 and 2, the project's steering committee decided two reductions of the electrical power supplies of the STYLED luminaires: after $T+6$ months measurements and before $T+18$ months measurements (see Table 2). The aim was to optimize luminance performance to the objectives set, with a corresponding reduction in luminaire power and energy consumption in accordance with normative requirements for luminance and uniformity factors. A graph of the average luminance of each section is shown in the Figure 4.

Table 2 - Theoretical and measured power consumption of the different sections.

\begin{tabular}{|c|c|c|c|c|}
\hline Sections & Theoretical & \multicolumn{3}{|c|}{ Measured power in W } \\
\cline { 3 - 5 } & $\begin{array}{c}\text { Measured } \\
\text { at T0 }\end{array}$ & $\begin{array}{c}\text { First reduction: after T6 } \\
\text { measurement }\end{array}$ & $\begin{array}{c}\text { Second reduction: before } \\
\text { T18 measurement }\end{array}$ \\
\hline S1: Lumiroute® 1 & $77 \mathrm{~W}$ & $78.7 \mathrm{~W}$ & $\begin{array}{c}62.6 \mathrm{~W} \\
(-20.5 \% \text { compared to T0) }\end{array}$ & $\begin{array}{c}50.9 \mathrm{~W} \\
(-35.6 \% \text { compared to T0) }\end{array}$ \\
\hline S2: Lumiroute $囚 2$ & $103 \mathrm{~W}$ & $103.2 \mathrm{~W}$ & $\begin{array}{c}80.4 \mathrm{~W} \\
(-22.1 \% \text { compared to T0) }\end{array}$ & $\begin{array}{c}67.3 \mathrm{~W} \\
(-34.8 \% \text { compared to T0) }\end{array}$ \\
\hline Section 3 and 4 & $140 \mathrm{~W}$ & \multicolumn{3}{|c|}{ 153W (no modification during the experiment) } \\
\hline
\end{tabular}




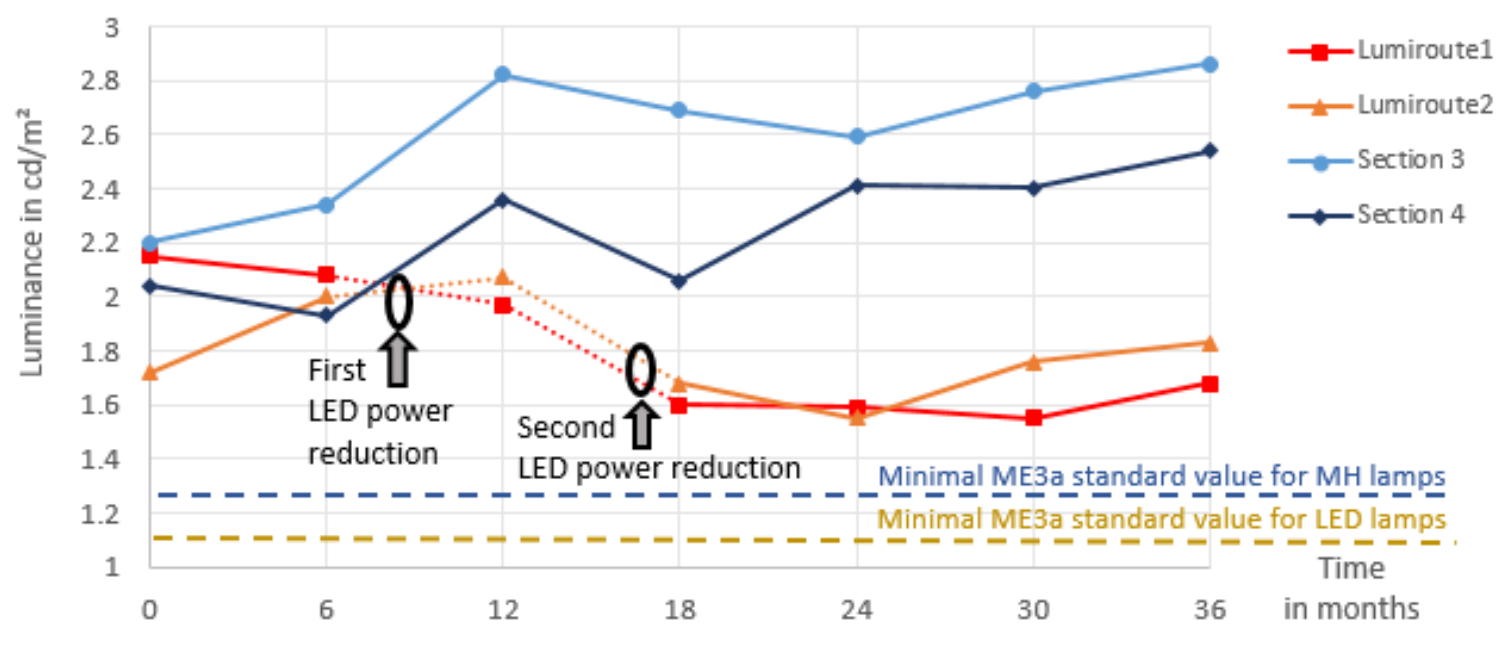

Figure 4 - Photometric measurements of the average luminance on each of the four sections.

The photometry measurements, done with CYCLOPE device, show the efficiency of the power adjustments performed on the STYLEDs (Lumiroute $® 1$ and Lumiroute $₫ 2$ sections) after $T+6$ and before $T+18$ months, which allowed the luminance performance to be adjusted as closely as possible to the class ME3a objective, in contrast to the control sections 3 and 4 , which have been increasingly overperforming since the beginning of the experiment (Figure 4). Throughout the experiment and for all sections, the overall uniformity was always above target values (Muzet et al., 2018). For the control sections, the increase in average luminance over the three years is important, suggesting that the increase in diffuse reflectance of the conventional road surface over time could compensate for the reduction in light output. It suggests that there was no need to apply a 0.8 maintenance factor in calculations for such conventional road surface and lighting. If the maintenance factor had been omitted in the initial dimensioning, then the $90 \mathrm{~W} \mathrm{MH}$ lamp would have been acceptable, resulting in less over-performance.

These results also demonstrate the efficiency of the LED power intensity adjustment setup, which allows the flux emitted by light sources to be adjusted as much as possible according to the evolutionary performance of the pavement surface to guarantee the required level of service throughout the experiment while controlling energy consumption.

\subsection{Measurement of the reflexion factor and evaluation of obtrusive light}

The reflection coefficient $\rho_{1}$ of the 4 pavements was measured during the experiment according to the CIE150 technical report (CIE, 2017). The results are presented in the Figure 5 and are consistent with the old LCPC measurements (CIE, 2017 annex E) with medium road surfaces at 0.09 and new road surfaces between 0.06 and 0.08 . For the treated sections 2 and 3 and the raw section $4, \rho_{1}$ has been constantly increasing since the beginning to 24 months and there is a stabilisation at the end of the experiment. The figure 5 also illustrates a much higher reflection coefficient $\rho_{1}$ for Lumiroute $® 1$, even at the end of the experiment. 


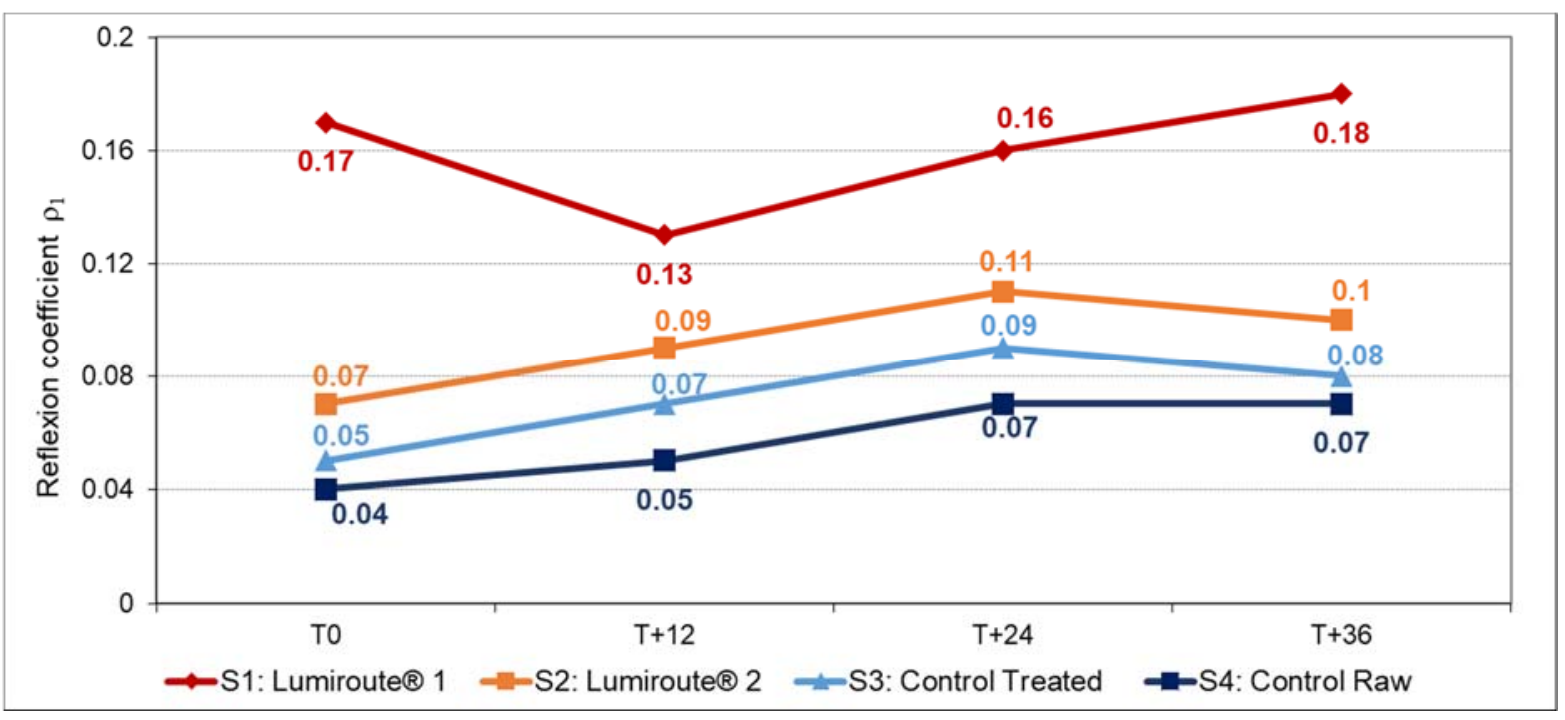

Figure 5 - Measurements of the reflection coefficient $\rho 1$.

The factors $U P F_{\max }$ and $U P F_{\min }$ and $R$ uL were computed according to (CIE, 2017) using the measured $\rho_{1}$ and a coefficient $\rho_{2}$ of the grassed sidewalk set at 0.2 . The results of the initial computations and after 36 months are presented in the Table 3. Even for the section with a higher reflection coefficient $\rho_{1}$ like Lumiroute ${ }^{\circledR} 1$ and 2 , with a lighting optimisation, the resulting UPF min (irreducible minimum flow reflected towards the sky reflected by the lighted surface) is lower than the control sections with no light optimisation.

At the end of the experiment, the evaluations of potentially lost flow UPF $F_{\max }$, show that the Lumiroute $\AA 1$ section has the lowest level of obstrusive light of the 4 sections, ahead of the Lumiroute $\circledast 2$ section $(+4 \%)$ and far ahead of sections $3(+130 \%)$ and $4(+123 \%)$.

It could also be noted that the ratio $R \cup L$ is always better for the Lumiroute $₫$ sections.

Table 3 - Calculation of factors $U P F_{\max }$ and $U P F_{\min }$ and $R \mathrm{UL}$ at T0 and T36 for the $\mathbf{4}$ sections.

\begin{tabular}{|l|c|c|c|c|c|c|c|c|}
\hline & \multicolumn{2}{|c|}{ S1: Lumiroute $\AA$ 1 } & \multicolumn{2}{c|}{ S2: Lumiroute $\AA$ 2 } & \multicolumn{2}{c|}{ S3: Control Treated } & \multicolumn{2}{c|}{ S4: Control Raw } \\
\hline Time in months & T0 & T36 & T0 & T36 & T0 & T36 & T0 & T36 \\
\hline Lamp Flux in lumen & 5800 & 3500 & 7500 & 4600 & 13200 & 13200 & 13200 & 13200 \\
\hline UPFmax in lumen & 1066 & 661 & 993 & 686 & 1384 & 1523 & 1337 & 1477 \\
\hline UPFmin in lumen & 530 & 351 & 273 & 234 & 293 & 468 & 234 & 410 \\
\hline ratio RuL & 2.0 & 1.9 & 3.6 & 2.9 & 4.7 & 3.3 & 5.7 & 3.6 \\
\hline
\end{tabular}

\subsection{Economic evaluation: energy assessment, investment and operating cost}

The annual energy consumption was calculated at the end of the experiment ( $T+36$ months), to be representative of the stable state of the road surface. These computations take into account the $40 \%$ power reductions applied on all the sections during the middle of the night. The Annual Energy Consumption Indicator (AECI or $D_{\mathrm{E}}$ ) has also been calculated in accordance with the European standard (CEN, 2015e), using the operational power $\mathrm{P} j$ of each period of operation $j$, t $j$, the corresponding operating time and $A$ the lighted surface.

$$
D_{E}=\frac{\sum_{j=1}^{m}\left(P_{j} \times t_{j}\right)}{A} \quad \text { in kWh.m }{ }^{-2}
$$

The results computed after 36 months are displayed on Table 4 . Lumiroute $\circledast$ sections are nearly 3 times less energy consuming than the control sections for an optimized service. 
Table 4 -Energy assessment after 3 years on the four sections.

\begin{tabular}{|l|c|c|}
\hline Sections & Annual consumption in kW h/year & $D_{\mathrm{E}}$ in $\mathrm{kW} \mathrm{h} / \mathrm{m}^{2}$ \\
\hline S1: Lumiroute ${ }^{2} 1$ & 1038 & 0.76 \\
\hline S2: Lumiroute ${ }^{\circledR} 2$ & 1335 & 0.98 \\
\hline S3: Control Treated & 3281 & 2.41 \\
\hline S4: Control Raw & 3286 & 2.41 \\
\hline
\end{tabular}

If, as suggested before, the 0.8 maintenance factor of the metal halide lamp was neglected, a $90 \mathrm{~W}$ metal halide lamp would have been sufficient to comply with the lighting class ME3a. The estimation of the resulting annual consumption would be $2187 \mathrm{kWh} /$ year instead of 3281 $\mathrm{kWh} /$ year for the installed $140 \mathrm{~W}$ lamp. With this hypothesis, Lumiroute ${ }^{8}$ section 1 is still 2 times less energy consuming than the control sections.

An economic evaluation was carried out for the 4 sections based on the cost of the work (pavement and lighting) and the operating costs. The investment costs were determined on surfaces with an equivalent surface area of $1300 \mathrm{~m}^{2}$. Operating costs have been calculated over 20 years and take into account both the electrical consumption of the installations and an estimate of their maintenance costs. They are compared to the 'before work' operating costs, when the lighting installation used HPS $400 \mathrm{~W}$ lamps. All these calculations, presented on Figure 6 , are specific to the experimental setup of this study.

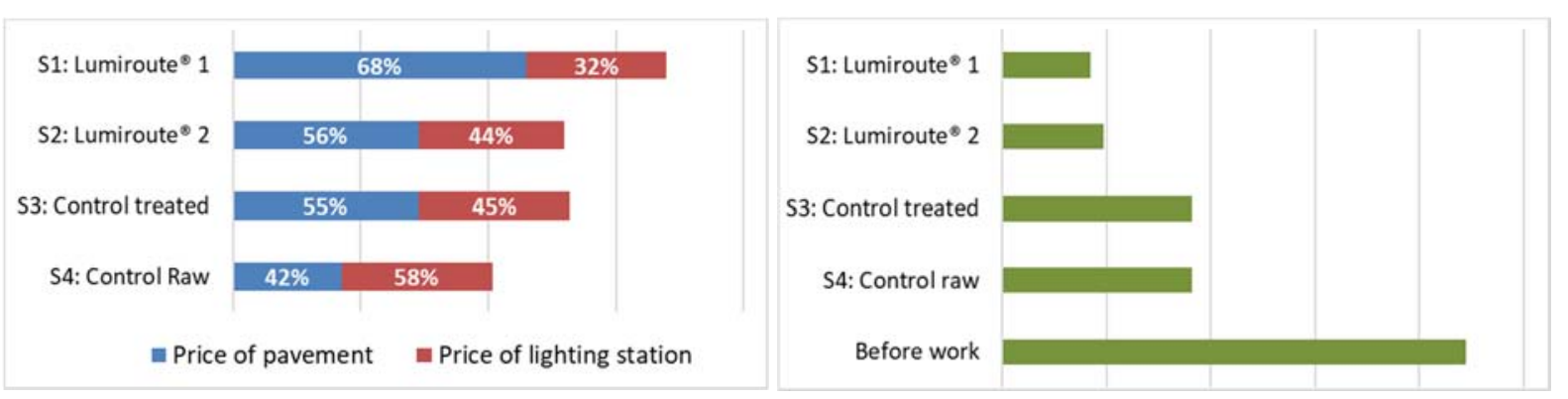

Figure 6 - Investment (left) and operating costs (right) for each sections. The 'Before work' corresponds to the operating costs before the renovation project.

Lumiroute $\circledast 1$ section has an important additional investment cost, compared to the other three sections. The Lumiroute ${ }^{\circ} 2$ and section 3 have a moderate cost (around $30 \%$ ) compared to the "raw" control section (section 4). The gain in terms of operating costs is significant, including for traditional sections with a gain of more than $50 \%$, and more than $65 \%$ for Lumiroute $\circledast$ sections. The payback period is 29 years for the Lumiroute ${ }^{\circledR} 1$ section and 14 years for the Lumiroute ${ }^{\circledR} 2$ section. In conclusion, the additional cost generated by Lumiroute $₫ 1$ coating remains important, resulting in a very long payback time, even in view of the initial energyintensive situation and despite a strong saving in operating costs. However, this technology could be used on limited areas due to its interesting performance. The Lumiroute ${ } 2$ process represents, on the other hand, a good compromise between the additional cost, the operating cost savings and the expected overall performance.

\section{Conclusions}

The on-site measurements of pavement photometry have confirmed the important initial heterogeneity of raw pavements. The water jet scrubbed sections offer interesting opportunities from the beginning because they have a low specularity and an initial homogeneity. Moreover, their increase of $Q_{0}$ with time is interesting because it could compensate for lamp ageing traditionally taken into account by the maintenance factor.

The collection of on-site photometric measurements will also be an input for the European SURFACE project (EMPIR, 2017), (Gidlund, 2019) which collects the r-tables of current and innovative road pavements across Europe in the context of a pre-normative study. 
The use of ILMD to characterise the photometric characteristics of the pavement was a success. It was possible to apply two reductions of the electrical power supplies of the LED luminaires, leading to more energy savings.

Generally speaking, the two Lumiroute ${ }^{\circledR}$ sections offer optimized performance and increased efficiency in comparison with the control sections, particularly with regard to light and energy performance and to the objective of controlling luminous flux. Compared to initial lighting installations before renovation (HPS 400W lamps), the savings in energy consumption are almost $90 \%$ for Lumiroute ${ }^{\circledR}$ coatings and $60 \%$ for control sections. The reflection coefficient $\rho_{1}$ of the 4 road surfaces was measured during the experiment. The two innovative pavements are systematically more reflective than conventional pavements. However, due to the use of

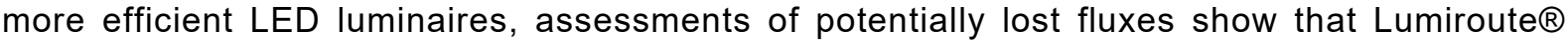
sections have significantly lower levels of obtrusive light than conventional sections $(+130 \%)$. However, the additional cost generated by the innovative sections was important, especially for the synthetic one. The section using a pavement with light aggregates and water jet scrubbing and adaptable LED luminaire represents a good compromise between a moderate additional cost, the operating cost savings and the expected overall performance.

The major outcomes of this project are:

- the very good results that can be obtained when considering both the road surface and the lighting fixture when designing a road with a lighting project,

- the importance of considering the real brightness and the specularity in the lighting project,

- the development of a diagnostic/adjustment process in this project, showing advantages for dynamic lighting fixtures, both in global flux and in spatial distribution.

\section{Aknowledgements}

We would like to thanks Patrick Tardieux and Philippe Chausse from Limoges Metropole, Aurelia Nicolai from Spie Batignolles Energie-Borja, Alexandre Taron from Zumtobel Group Lighting France, Cyril Chain, Philippe Carle, Marc Toinette and Fabrice Fournela from Cerema.

\section{Fundings}

This project received financial support of the MTES (French Ministry of the Ecological Transition and Solidarity) for the coordination and the technical evaluation of the Lumiroute $₫$ project.

Part of this work received funding from the EMPIR programme project "16NRM02 Surface Pavement surface characterisation for smart and efficient road lighting". EMPIR programme is co-financed by the Participating States and from the European Union's Horizon 2020 research and innovation program.

\section{References}

CEN 2003. CEN 13201-2:2003. Road lighting - Part 2: Performance requirements.

CEN 2009. CEN/TS 15901-6:2009. Road and airfield surface characteristics - Part 6: Procedure for determining the skid resistance of a pavement surface by measurement of the sideway force coefficient (SFCS): SCRIM®

CEN 2010. CEN 13036-1. Road and air field surface characteristics - Test methods. Part 1: Measurement of pavement surface macrotexture depth using a volumetric patch technique.

CEN 2015a. CEN 13201-1:2015. Road lighting - Part 1: Selection of lighting classes.

CEN 2015b. CEN 13201-2:2015. Road lighting - Part 2: Performance requirements.

CEN 2015c. CEN 13201-3:2015. Road lighting - Part 3: Calculation of performance.

CEN 2015d. CEN 13201-4:2015. Road lighting - Part 4: Methods of measuring light performance. 
CEN 2015e. CEN 13201-5:2015. Road lighting - Part 5: Energy performance indicators.

CHAIN C, LOPEZ F AND VERNY P. 2007. Impact of real road photometry on public lighting design. In: 26th session of the CIE, Beijing, China.

CHAIN, C., VERNY, P., MUZET, V., GREFFIER, F. 2015. Optimization of road surfaces reflection properties and lighting., In: 28th session of the CIE, Manchester, United Kingdom.

CIE 1984. CIE 066-1984. Road Surfaces and lighting, joint CIE/PIARC publication.

CIE 1993. CIE 093-1993. Guide for floodlighting.

CIE 2001. CIE 144-2001. Road surface and road marking reflexion characteristics, Technical Report.

CIE 2019. CIE 140:2019. Road Lighting Calculations, 2nd Edition. Vienna: CIE.

DUMONT, E. 2007a. Photométrie des chaussées et éclairage public, In : Etudes et Recherches des laboratoires des ponts et chaussées, CR45, LCPC (eds), p.82 (in french).

DUMONT, E., PAUMIER, J.L. 2007b. Are standard tables R still representative of the properties of road surfaces in France? In: 26th session of the CIE, Beijing, China.

EMPIR 2017. 16NRM02 Surface Pavement surface characterisation for smart and efficient road lighting, European EMPIR project 2017-2020, http://surface-nrm02.eu/.

GIDLUND, H., LINDGREN, M., MUZET, V., ROSSI G, IACOMUSSI, P. 2019. Road surface photometric characterisation and its impact on energy savings. Coatings, 9(5), 286; https://doi.org/10.3390/coatings9050286.

GREFFIER, F., CHARBONNIER, P., TAREL, J.P., et al. 2015. An automatic system for measuring road and tunnel lighting performance. In: 28th session of the CIE, Manchester, United Kingdom, 2015.

JACKETT, M., FRITH, W. 2010. Reflection properties of New Zealand road surfaces for road lighting design. In: IPENZ Transportation Group Conference, Christchurch, New Zealand, 14p.

MUZET, V., PAUMIER, J.L., GUILLARD, Y. 2007. COLUROUTE: a mobile gonio-reflectometer to characterize the road surface photometry. In: CIE international symposium on road surface characteristics, Torino, Italy.

MUZET, V., ABDO, J. 2017. On site photometric characterisation of concrete pavements with COLUROUTE device. In: Lux Europa, Ljubljana, Slovenia, pp.164-168.

MUZET, V., GREFFIER, F., NICOLAÏ, A., TARON, A., VERNY, P. 2018. Evaluation of the performance of an optimized road surface/lighting combination. Lighting Research and Technology, first published on line, DOI: 10.1177/1477153518808334

PAUMIER, J.L., LEGOUEIX, G., DUPONT, P., et al. 2006. Propriétés photométriques des revêtements de chaussée. CFTR-Info, 14, 12p (in french).

PETRINSKA, D., IVANOV, D., DAVLOV, D., et al. 2015. Road surface reflection properties of typical for Bulgaria pavement materials, In: Lux Junior, Dörnfeld, Germany.

YLINEN, A., PUOLAKKA, M., HALONEN, L. 2010. Road surface reflection properties and applicability of the r-tables for today's pavement material in Finland, Light and Engineering, 18(1): 78-90.

VAN BOMMEL, W. 2015. Road Lighting: Fundamentals, Technology and Application, In: Springer International Publishing Switzerland (eds), p.334. 